\title{
Texture Classification using Information Theory
}

\author{
Hayit K. Greenspan and Rodney M. Goodman \\ Department of Electrical Engineering, 116-81 \\ California Institute of Technology \\ Pasadena, CA 91125 \\ USA
}

Visual texture is one of the most fundamental properties of a visible surface. It participates as one of the major modalities which help us in the understanding of our visual environment. The different textures in an image are usually very apparent to a human observer, but automatic description of these patterns has proved to be complex.

The framework for the proposed texture analysis task consists of two major stages. The first stage performs feature extraction by transforming the image space into an array of $\mathrm{N}$-dimensional feature vectors. The second stage analyzes the $\mathrm{N}$-dimensional feature space. Clusters are found via "unsupervised learning" and following that, a labeling process, based on previous experience or knowledge, enables the recognition to be performed via a "supervised learning" stage.

There is much evidence supporting the use of Gabor-like filters in the feature-extraction phase [1]. The features thus extracted are in the orientation and frequency domains. An open question is what filters are to be utilized for the feature extraction, i.e. what minimal number of frequencies and orientations are representative of the textures and contain sufficient information for the texture labeling. In the proposed approach we reduce the $\mathrm{N}$-dimensional feature space both in the unsupervised and supervised stages of analysis using information theoretic criteria.

In the unsupervised stage a Neural-Network Vector Quantization procedure is used based on the Kohonen topology-preserving mapping [2]. This procedure enables quantization of the continuous valued features into $M$ discrete levels and the projection of the $\mathrm{N}$-dimensional space into a lower dimension clustered map, for initial segmentation of the textured image. A supervised stage follows. The goal of the supervised stage is to utilize the existing information in the feature maps for higher level analysis, such as input labeling and classification. In particular we need to learn a classifier which maps the output features of the Kohonen stage to the texture class labels. We utilize a rule-based information theoretic approach which is an extension of a first order Bayesian classifier, because of its ability to output probability estimates for the output classes [3]. The classifier defines correlations between input features and output classes as probabilistic rules of the form: If
$Y=y$ then $X=x$ with prob. $P$. A data driven supervised learning approach utilizes an information theoretic measure to learn the most informative links or rules between features and class labels [4]. The classifier then uses these links to provide an estimate of the probability of a given output class being true. When presented with a new input evidence vector, a set of rules $R$ can be considered to "fire". The classifier estimates the posterior probability of each class given the rules that fire in the form $\log (p(x) / R)$, and the largest estimate is chosen as the class label decision.

The performance of the presented framework is tested on both synthetic and natural textures. A correct classification rate of $95-99 \%$ is obtained for the texton textures. A slightly lower percentage is obtained for the natural texture mosaics. In addition to the labeling maps, the rule-based information theoretic approach enables us to output probability estimates for the output classes, thus adding confidence measures to our decisions. The actual class probabilities could be used for further higher level processing such as a feedback loop in the suggested framework. It is apparent from the high percentage classification rate that the information criteria used, both in the unsupervised and supervised stages, have succeeded in capturing an informative minimal representation of the input domain.

\section{References}

[1] J. Malik and P. Perona, "Preattentive texture discrimination with early vision mechanisms," Journal of Optical Society of America, A, May 1990.

[2] T. Kohonen, "Self Organisation and Associative Memory," Springer-Verlag, 1984.

[3] R.M. Goodman, C. Higgins, J. Miller and P. Smyth, "A Rule-Based Approach to Neural Network Classifiers," INNC 90 Paris - International Neural Network Conference, Palais Des Congres, Paris, France, July 9-13, 1990.

[4] R.M. Goodman and P. Smyth, "The Induction of Probabilistic Rule Sets - The ITRULE Algorithm," Proceedings of the 1989 International Workshop on Machine Learning, San Mateo, CA: Morgan Kaufmann, pp. 129-132, 1989.

This work is supported in part by the Army Research Office under contract No. DAAL03-89-K-0126, and in part by DARPA under contract no. AFOSR-90-0199. 